\title{
Advanced Signal Processing Methods for Pulsed Laser
}

\section{Vibrometry}

\author{
Julien Totems, ${ }^{1, *}$ Véronique Jolivet, ${ }^{1}$ Jean-Philippe Ovarlez, ${ }^{1}$ and Nadine Martin ${ }^{2}$ \\ ${ }^{\text {I}}$ Onera, The French Aerospace Lab, Theoretical and Applied Optics Department, Chemin de la \\ Hunière, 91761 Palaiseau Cedex, France \\ ${ }^{2}$ Gipsa-Lab, 961 rue de la Houille Blanche, Domaine Universitaire BP 46, F-38402 Saint- \\ Martin d'Hères, France \\ *Corresponding author: julien.totems@onera.fr
}

Although pulsed coherent laser radar vibrometry has been introduced as an improvement over its Continuous Wave (CW) counterpart, it remains very sensitive to decorrelation noises such as speckle, and other disturbances of the measurement. Taking advantage of more polyvalent poly-pulse waveforms, we address the issue with advanced signal processing. We have conducted what we believe is the first extensive comparison of processing techniques considering $\mathrm{CW}$, pulse-pair and poly-pulse emissions. In this framework, we introduce a computationally efficient maximum likelihood estimator and test signal tracking on pseudo time-frequency representations, which respectively help deal with speckle noise and fading of the signal, in harsh noise conditions. Our comparison on simulated signals is validated on a $1.55 \mu \mathrm{m}$ all-fiber vibrometer experiment, with an apparatus simulating vibration and strong speckle noise. Results show the advantage of estimators taking into account actual noise statistics, and call for a wider use of timefrequency representations to track the vibration modulated signal. 
OCIS codes: $280.0280,280.3340,120.7280$.

\section{Introduction}

Coherent laser radars (lidars) are able to sense the small amplitude vibrations of remote surfaces thanks to the Doppler effect. Long range laser Doppler vibrometry has been successfully applied to an increasing number of situations where the interest is to determine the vibration characteristics of an inaccessible target. These applications range from the structural assessment of potentially damaged buildings [1] to target identification for military purpose [2,3]. Although vibration frequencies and velocities are very different in magnitude, in both cases the aim is to identify the modal frequencies of the target.

The measurement is performed as shown on Fig. 1. First, coherent heterodyne detection, done by mixing the Doppler-shifted backscattered laser wave and part of the emission (Local Oscillator, LO) on a detector, is the source of a heterodyne signal with an Instantaneous Frequency (IF) linked to the instantaneous velocity of the target surface. This heterodyne signal is frequency demodulated to retrieve the vibration velocity time series. Then, the velocity time series is Fourier transformed and a vibration spectrum is obtained, which is analyzed to extract vibrational features. Interesting features include the modal frequencies of the target, appearing as peaks on the spectrum if the Signal-to-Noise Ratio (SNR), between the peak Power Spectral Density (PSD) and the noise floor power spectral density, is high enough.

In addition to continuous emission, a pulsed emission has been considered [3]. The simplest and most usual waveform is the pulse-pair, inherited from meteorological radars, processed by estimating the phase shift between the two consecutive pulses. At reception, each waveform provides one velocity sample of the time series. Poly-pulses were also tested [4]. 
Pulsed vibrometry directly benefits from existing techniques in radar, for waveform design as well as signal processing.

The advantages of pulsed vibrometry have been discussed in [5,6]. First, a monostatic configuration of the vibrometer is easier to implement with static targets, because returns from the target can be temporally separated from parasitic reflections on the optics; having a single collimating optic in the instrument eases its pointing and focusing, and might be required for compactness. Also, simultaneous telemetry by time-of-flight measurement becomes possible, allowing a time-gating of the reception and the separation of multiple targets in the laser beam. Finally, higher peak power is available for the same average laser power, resulting in higher signal detectability, and even slightly more accurate instantaneous Doppler shift estimation at very long range.

Yet, as was shown by the comparison of $\mathrm{CW}$ and pulse-pair operation modes led by Hill et al. [6], the latter suffers from several drawbacks. When compared with the same mean emitted laser power, in normal noise conditions, pulse-pair does not perform better than $\mathrm{CW}$ in obtaining good SNR on the final vibration spectrum. Each pulse-pair can yield slightly more accurate velocity estimates at long range thanks to a higher peak power, but with considerable averaging over numerous samples, CW mode still gets better results. Furthermore, strong phase noise, originating from speckle or laser phase noise, greatly decreases the pulsed mode performance, and pulse-pairs have an unambiguous velocity range only a few times larger than their velocity resolution.

In summary, past studies have concluded that, despite its unique potential for very long range, multifunction and monostatic instruments, pulsed vibrometry is impaired by its sensitivity to the disturbances of the measurement channel. However, the wide diversity of waveforms and 
signal processing available, compared to simple pulse-pair operation, indicates that performance improvement is possible. In this article, we investigate the potential of advanced signal processing techniques, applied to the poly-pulse waveforms already introduced in [4], which benefit from supplementary degrees of freedom. In the unfavorable conditions of strong speckle noise, we conduct an extensive performance comparison between $\mathrm{CW}$, pulse-pair and poly-pulse operation, processed by various classical and new processing techniques. The objective is to show how and to what extent pulsed vibrometry can be made more robust to harsh noise conditions.

Section 2 introduces signal model and hypothesis. The characteristics of poly-pulse waveforms and known signal processing methods for $\mathrm{CW}$ and pulsed mode are recalled in Section 3. In section 4, we propose and qualify new signal processing techniques for poly-pulse waveforms. We compare them to existing techniques, for the various waveforms, through simulation in section 5. An experimental validation of this comparison, using an all-fiber vibrometer, is presented in section 6 . Section 7 concludes.

\section{Signal model}

The photocurrent, downshifted from the carrier frequency $\mathrm{f}_{0}$ to the null frequency, either analogically (I/Q demodulation) or digitally, is expressed, in a complex form:

$$
i_{S}(t)=\mu(t) \cdot i_{h e t}(t)+i_{b}(t)=\mu(t) \cdot i_{0} \cdot m(t) \cdot \exp \left(j \varphi_{v i b}(t)\right)+i_{b}(t)
$$

where $\mu(t)$ is the amplitude modulation applied to the laser emission in pulsed mode $(\mu(t)=1$ in CW mode), $\mathrm{i}_{\text {het }}(\mathrm{t})$ is the heterodyne current, with a mean amplitude $\mathrm{i}_{0}, \mathrm{~m}(\mathrm{t})$ is a complex multiplicative noise, circular and centered, with a variance set to $1, \varphi_{\text {vib }}(\mathrm{t})=4 \pi \cdot \mathrm{X}_{\mathrm{vib}}(\mathrm{t}) / \lambda$ is the phase modulation for the laser wavelength $\lambda(1.55 \mu \mathrm{m})$ caused by $\mathrm{x}_{\mathrm{vib}}(\mathrm{t})$, the targeted surface 
vibration displacement projected along the laser line of sight. In case of a sinusoidal vibration, $\mathrm{x}_{\mathrm{vib}}(\mathrm{t})=\mathrm{a} \sin \left(2 \pi \mathrm{f}_{\mathrm{vib} \cdot} \mathrm{t}+\varphi\right)$ and $\mathrm{v}_{\mathrm{vib}}(\mathrm{t})=2 \pi \mathrm{af} \mathrm{v}_{\mathrm{vib}} \cos \left(2 \pi \mathrm{f}_{\mathrm{vib} \cdot \mathrm{t}} \mathrm{t}+\varphi\right)=\mathrm{V}_{\max } \cos \left(2 \pi \mathrm{f}_{\mathrm{vib}} \cdot \mathrm{t}+\varphi\right)$. Lastly, $\mathrm{i}_{\mathrm{b}}(\mathrm{t})$ is an additive complex noise (detector and photon noise), white, Gaussian valued, circular and centered, with variance $\sigma_{\mathrm{b}}{ }^{2}$. The time-averaged Carrier-to-Noise Ratio (CNR) is defined as $\langle\mathrm{CNR}\rangle=\left\langle\left|\mathrm{i}_{\text {het }}\right|^{2}\right\rangle \mid\left\langle\left|\mathrm{i}_{\mathrm{b}}\right|^{2}\right\rangle=\mathrm{i}_{0}^{2} / 2 \sigma_{\mathrm{b}}^{2}$.

Complex multiplicative noise $\mathrm{m}(\mathrm{t})$ contains amplitude and phase fluctuations terms, and is the result of several phenomena [7]: speckle noise, laser phase noise, and the effect of atmospheric turbulence.

The random speckle pattern backscattered by the target is not static if the illuminated surface is moving, and as a consequence the amplitude and phase of the received wave will vary, a phenomenon either called "speckle noise" or "target decorrelation noise" in literature [8]. The resulting multiplier has a complex normal distribution and autocorrelation function $\Gamma(\tau)=\exp (-$ $\left.\tau^{2} / \tau_{\mathrm{c}}{ }^{2}\right)=\exp \left(-\mathrm{B}_{\text {speckle }}{ }^{2} \tau^{2}\right)$. This means its phase and amplitude is roughly stable over durations shorter than $\tau_{\mathrm{c}}=1 / \mathrm{B}_{\text {speckle }}$, and that its frequency width is about $2 \mathrm{~B}_{\text {speckle }} / \pi$. Depending on target distance and movement (target rotation has the largest impact), speckle bandwidth $\mathrm{B}_{\text {speckle }}$ varies in the $100 \mathrm{~Hz}-100 \mathrm{kHz}$ range.

Laser phase noise is due to the finite spectral linewidth of the laser: as the emitted frequency is random, the frequency spread of the beat signal between the received wave and the local oscillator increases as the target is further away and the waves become decorrelated. The result is a random phase term, which PSD can be predicted given the optical path difference. It can however be greatly mitigated by using a well chosen delay line in the local oscillator path.

Last, atmospheric turbulence, as an index distribution pushed across the beam by transverse wind, can also produce a complex multiplicative noise that affects both phase and 
amplitude, which characteristics have been inferred by Ishimaru [9]. Yet, the phase term due to turbulence piston is rather slow. Unless the laser beam is low above the ground, the amplitude fluctuations are slower than those due to target speckle, and turbulence noise is negligible in applications that involve moving targets.

Complex multiplicative noise impacts the measurement through signal fading (temporarily low CNR), as well as spectral broadening because of phase fluctuations, which directly lowers the accuracy of the velocity estimation.

The signal model of Eq. 1 is based on several simplifying hypothesis: i) it is assumed that any bulk Doppler shift due to target global velocity has previously been removed; ii) the illuminated surface vibrates as a whole, and no separate vibrators generate signals with various IFs; iii) the target's distance is known precisely (by means of simultaneous telemetry); iv) the emitted mean laser power is equal for all operating modes, which implies taking $\left.\left\langle\mu(t)^{2}\right\rangle=1 ; v\right)$ lastly, we neglect the phase effects of atmospheric turbulence and laser phase noise and only consider speckle noise.

In that case, the noise parameters are the speckle bandwidth $\mathrm{B}_{\text {speckle }}$ and the CNR. $\mathrm{B}_{\text {speckle }}$ is set to $5 \mathrm{kHz}$ (as induced by the parallax of a target with a $500 \mathrm{~km} / \mathrm{h}$ velocity perpendicular to the line of sight of a laser radar with $100 \mathrm{~mm}$ pupil). The vibration amplitude is chosen so that the modulation bandwidth is of the same order. Such values are consistent with the actual parameters expected for long range moving vehicle identification (previous studies $[6,8]$ considered speckle noise with lower bandwidth, few hundred Hertz). The CNR, averaged in time, is a common parameter for all operating modes, given the hypothesis of equal mean laser power in every case. It is calculated in the full sampling band of $1 \mathrm{MHz}$.

\section{Background}




\subsection{Poly-pulse waveforms}

Pulsed waveforms are created by modulating the photocurrent amplitude by a square wave $\mu(t)$.

Because of the poor ambiguity-to-resolution ratio (i.e. measurement dynamic) offered by pulse-pair waveforms, we considered more general poly-pulse waveforms as was already done by Gatt et al. [4]. Though staggered poly-pulses present the highest measurement dynamic in theory, their velocity ambiguities are difficult to solve in harsh noise conditions, and we rather focus on regular poly-pulses. As shown in Fig. 2, we define poly-pulses as a finite succession of $\mathrm{N}_{\mathrm{p}}$ pulses of short duration $\mathrm{t}_{\mathrm{p}}$. These trains of pulses are repeated with period T, i.e. at repetition frequency $\mathrm{PRF}=1 / \mathrm{T}$. The pulses in the train are equally separated by duration $\mathrm{T}_{\mathrm{S}}$, and the total waveform duration is $\mathrm{T}_{\mathrm{m}}=\left(\mathrm{N}_{\mathrm{p}}-1\right) \cdot \mathrm{T}_{\mathrm{S}}$.

We now discuss the constraints that apply on waveform parameters, in order to choose a waveform best suited to given vibration and noise conditions.

One Instantaneous Frequency (IF) estimation (i.e. a velocity estimate) is performed for each poly-pulse. Its general principle is to differentiate the phase of singular pulses along the train, in order to obtain the IF, which is supposed constant during the short waveform duration $\mathrm{T}_{\mathrm{m}}$, chosen accordingly. The precision on this estimation is Fourier limited at approximately $1 / \mathrm{T}_{\mathrm{m}}$, and, as a consequence, longer waveforms allow better precision. But, when compared to a continuous wave of same effective duration, a poly-pulse waveform has the advantage that the energy is concentrated in pulses, and the signal can be temporally separated from most of the noise affecting the measurement. In case of strong additive noise, with the same energy, slightly better velocity precision is obtained in pulsed mode. The downside is that Doppler ambiguities exist: velocity is known only within ambiguity interval $V_{a}=\lambda\left(2 T_{S}\right)$. 
The constraints for waveform design are the following: i) the waveform repetition frequency PRF has to match Nyquist's criterion for the correct sampling of the vibration itself, PRF $>2 f_{\text {vib,max }}$, with $f_{\text {vib,max }}$ is the maximum significant vibration frequency of the target. ii) $T_{S}$ should be a function of the maximum velocity $\mathrm{V}_{\max }$, to remain in ambiguity interval $\mathrm{V}_{\mathrm{a}}$ : $\mathrm{T}_{\mathrm{S}} \leq \lambda /(4$ $\mathrm{V}_{\max }$ ). iii) depending on the required velocity accuracy, $\mathrm{N}_{\mathrm{p}}$ should then be chosen so that the effective duration of the waveform $\sigma_{t}=\int t^{2} \cdot \mu(t) d t$, roughly proportional to $\mathrm{N}_{\mathrm{p}}$, allows a small enough velocity resolution $\delta \mathrm{V}=\lambda / 8 \pi \sigma_{\mathrm{t}}$, while satisfying the hypothesis of a stationary IF during the waveform duration. Poly-pulses indeed provide a larger measurement dynamic (linear) $\mathrm{D}=\mathrm{V}_{\mathrm{a}} / \delta \mathrm{V}$ than pulse-pairs: $\mathrm{D}_{2 \text { pulses }} \approx 9$, while $\mathrm{D}_{4 \text { pulses }} \approx 28$, for instance.

Those three fundamental constraints are set according to the expected vibration, for which coarse assumptions can be made by knowing the nature of the target. Beyond this essential adaptation to the vibration, actually optimizing waveform parameters will rely on other variables, such as the noise conditions. In fact, the optimal pulse number $\mathrm{N}_{\mathrm{p}}$ is very dependent on the relative power of the signal and noise, as well as the correlation time of the phase of the signal, due to phase noise. Indeed, the duration of the poly-pulse should remain shorter than this correlation time. Like in [6], we choose to compare waveforms by setting the same mean laser power for all, in order to avoid taking into account the evolving limitations of lasers, and benefit from a common ground to evaluate the efficiency in spending a given energy for the measurement. This hypothesis implies that, this energy being equally divided into the $\mathrm{N}_{\mathrm{p}}$ individual pulses, their peak power decreases as $\mathrm{N}_{\mathrm{p}}$ is increased. Simple considerations led us to choose $\mathrm{N}_{\mathrm{p}}=6$ (above which velocity resolution stalls because of IF non-stationarity) and $\mathrm{PRF}=500 \mathrm{~Hz}$ (just above Nyquist's criterion for a $200 \mathrm{~Hz}$ maximum vibration frequency) in our study. 


\subsection{Signal processing for coherent laser radar vibrometry}

In this sub-section, we recall signal processing strategies for vibrometry and present the estimators that are compared in section 6.

The goal of the measurement is to determine the vibration velocity by IF estimation, in order to evaluate modal frequencies of vibration, whereas detection noise, phase noise and fading disturb this estimation. Two main strategies stand out: i) phase differentiation over samples (in $\mathrm{CW}$ ) or pulses, like in conventional frequency demodulation, ii) spectral maximum estimation or Time-Frequency processing, which works by determining the frequency localization of the maximum of energy along time, on a Time-Frequency Representation (TFR) such as a spectrogram [10]. Parametric estimation, based on a full signal model, is a third possible way, supposedly optimal $[6,11]$ but never applied because of its complexity. We have not considered this approach in this paper.

The vibration velocity is usually Fourier transformed to identify peaks at potential modal frequencies, which are the actual data of interest. A relevant performance indicator is then the SNR, the power spectral density ratio between the peak and the noise spectrum. For completeness, we mention there are variations for this last step of the process, such as the parametric estimation of the vibration modes given the pre-evaluated noise variance [12].

\section{IF estimators in $C W$ mode}

The sliding « coherent average » of phase differences between $\mathrm{K}$ consecutive samples is an enhancement of pulse-pair processing [6]. This estimator, first in Tab. 1, belongs to the phase differentiation family defined earlier and is called Autocorrelation First Lag (AFL). 
The following two techniques use a TFR; the spectrogram is here preferred to more performing kernels such as Born-Jordan's [11], because of its much shorter computation time. First, we implement the centroid of the spectrogram columns [7], with a circular sum in order to avoid bias from the non-zero-mean noise background (SpectroGram Centroid, SGC). But the spectrogram can also be processed using a spectral equivalent of the phase based estimator originally given by Lee, as explained in $[13,14]$. Lee's Spectral Matching (LSM) consists in finding the best match in position between a reference spectrum $S_{\text {ref }}(f)$ (Gaussian spectrum induced by the speckle noise) and the short-term spectra composing the spectrogram.

Centroid based (SGC) and Lee's (LSM) estimators allow a large improvement over the simple maximum detection along time on a Time-Frequency Representation, especially when strong complex multiplicative noise is involved, and compared to other frequency estimators [10, 14].

\section{IF estimators in pulsed mode}

In Pulse-Pair mode, the fourth estimator of Tab. 1 uses the phase difference between each pair of pulses (Pulse-Pair estimator, PP). Telemetry data or previously performed pulse detection is necessary in order to properly window the signal. This method is straightforwardly extended to Poly-Pulse waveforms: the phase difference between consecutive pulses is coherently averaged (Poly Pulse-Pair estimator, PPP). This estimator is the fastest applied to poly-pulses. But several authors, as in $[14,15]$, insist on the benefit of phase differentiation over non-consecutive pulses.

The Autocorrelation Fourier Transform (AFT) estimator thus performs a linear regression of the autocorrelation function phase, i.e. the search of the Fourier Transform (FT) maximum, after a proper windowing. In order to take into account the decorrelation induced by speckle 
noise, and to avoid using uncorrelated pairs of pulses, windowing function $h(\tau)$ is set to the modulus of $\Gamma_{\mathrm{is}, \mathrm{k}}(\tau)$, averaged on all poly-pulses.

The matched filter approach, as in radar processing, equivalent to the previous one when $h(\tau)=1$, uses the spectrum of the received waveform multiplied by the emitted waveform (Matched Filter, MF). It can be applied to any waveform. With only additive white noise, this estimator is the maximum likelihood estimator, asymptotically optimal at high CNR.

\section{Advanced IF estimation with poly-pulse waveforms}

\subsection{Maximum likelihood IF estimation}

In coherent laser radar, however, multiplicative noise is also present. We thus propose the actual maximum likelihood estimator of the instantaneous frequency of a received poly-pulse, given the signal model of Eq. 1. We assume stationary waveforms and prior knowledge of the noise parameters.

For faster computation, the likelihood is calculated by applying a variable change proposed by Ghogho et al. in [16]. It uses the fact that if the phase of the signal can be put in factor, what remains is the sum of multiplicative and white additive noises, which have known statistics. The resulting poly-pulse instantaneous frequency Maximum Likelihood (ML) estimator is

$$
\hat{f}_{\text {inst }}(t=k / P R F)=\underset{f}{\arg \max }\left(-\operatorname{Re}\left(\vec{s}^{\prime}\right)^{T} \cdot Q_{s^{\prime}}{ }^{-1} \cdot \operatorname{Re}\left(\vec{s}^{\prime}\right)-\operatorname{Im}\left(\vec{s}^{\prime}\right)^{T} \cdot Q_{s^{\prime}}{ }^{-1} \cdot \operatorname{Im}\left(\vec{s}^{\prime}\right)\right)
$$

with

$$
\vec{s}^{\prime}(p)=<i_{s}>_{k, p} \exp \left(-j 2 \pi f \cdot p T_{S}\right) \text { for } \mathrm{p}=1, \ldots, \mathrm{N}_{\mathrm{p}} \text { and } Q_{s^{\prime}}=C N R_{p e a k} Q_{m}+I_{N_{p}}
$$


where $\mathrm{Q}_{\mathrm{s}}$, is the sum of the noise covariance matrices, as sampled by the pulses, $\mathrm{Q}_{\mathrm{m}}$ is the covariance matrix of multiplicative noise $\mathrm{m}$, and $\mathrm{I}_{\mathrm{Np}}$ is the identity matrix. Element $(\mathrm{p}, \mathrm{q})$ of $\mathrm{Q}_{\mathrm{m}}$ is given by the autocorrelation function of the considered multiplicative noise, in our case speckle:

$$
Q_{m}(p, q)=Q_{\text {speckle }}(p, q)=\Gamma_{\text {speckle }}\left((p-q) T_{S}\right)=e^{-B_{\text {speckl }}^{2} T_{S}^{2}(p-q)^{2}} \text { for } \mathrm{p}, \mathrm{q}=1, \ldots, \mathrm{N}_{\mathrm{p}}
$$

ML is the equivalent of Levin's own maximum likelihood estimator developed for CW laser radar, explained in [13], but applied to poly-pulse waveforms. As Levin's estimator does in the spectral domain, when the phase modulation of the signal is suppressed assuming the instantaneous frequency was $\mathrm{f}$, this likelihood quantifies how much the result is close to having the same covariance matrix as the supposedly remaining multiplicative and additive noises. Like the matched filter, it can also been seen as a linear regression of the phase: the scalar product with $\exp (-\mathrm{j} 2 \pi \mathrm{ft})$ is calculated on series of correlated pulses selected by $\mathrm{Q}_{\mathrm{s}}{ }^{-1}$, and averaged over the considered series. Note that in fact, when $B_{\text {speckle }}$ tends towards zero, the ML estimator becomes equivalent to the matched filter.

The maximum likelihood is theoretically optimal if the signal model of Eq. 1 is verified, but might not perform as well under strong noise conditions (low CNR or very high $\mathrm{B}_{\text {speckle }}$ ), or in case of deviations from the model. Also, the knowledge of noise parameters CNR and $\mathrm{B}_{\text {speckle }}$ is necessary to use this estimator. Both of them can be evaluated by studying the amplitude of the signal: CNR via the ratio of power in and out of the pulses, and $\mathrm{B}_{\text {speckle }}$ by calculating the width of the autocorrelation function of amplitude fluctuations. A third, more direct method is to evaluate $\mathrm{Q}_{\mathrm{s}}$, from the signal, demodulated by a quick coarse IF estimate. This can be written

$$
\hat{Q}_{s^{\prime}}=\sum_{\wedge k=1}^{K} \vec{s}_{k}^{\prime} \cdot \vec{s}_{k}^{\prime t} \text { with } \vec{s}_{k}^{\prime}(p)=<i_{s}>_{k, p} \exp \left(-j 2 \pi \hat{f}_{k} \cdot p T_{S}\right) \text { for } \mathrm{p}=1, \ldots, \mathrm{N}_{\mathrm{p}}
$$


where $\mathrm{K}$ is the total number of waveforms during the measurement, and $\mathrm{f}_{\mathrm{k}}$ is the coarse estimate previously obtained for waveform \#k. The PPP estimator is fast enough to provide this estimate.

The proposed ML estimator can be modified in case the noise characteristics differ from those of our primary model; for instance, if there is laser frequency noise in addition to the speckle noise in $\mathrm{m}(\mathrm{t})$, then $\mathrm{Q}_{\mathrm{m}}$ becomes the element-wise product of $\mathrm{Q}_{\text {speckle }}$ and $\mathrm{Q}_{\text {laser }}$, also given by the autocorrelation function of the laser phase noise, found in [17].

\subsection{Performance of the $M L$ estimator}

In order to assess the performance of the ML estimator, the Cramér-Rao Bound (CRB) of the velocity estimation over each received poly-pulse waveform is calculated from the likelihood function, without variable change and pulse average:

$$
\sigma_{v} \geq \frac{\lambda}{2}\left[\operatorname{Tr}\left(\left(\frac{\partial Q}{\partial f} Q^{-1}\right)^{2}\right)\right]^{-1 / 2}
$$

in which $\operatorname{Tr}()$ designates the matrix trace operation and $\mathrm{Q}$ is the covariance matrix of the nonzero samples of the signal, with elements

$$
Q(u, v)=\mu_{\max }^{2} i_{0}^{2} \Gamma_{m}((u-v) \Delta t) \cdot e^{j 2 \pi f(u-v) \Delta t}+\sigma_{b}^{2} \delta(u-v)
$$

for $\mathrm{u}, \mathrm{v}=1, \ldots, \mathrm{N}_{\mathrm{w}}$ so that $\mu(\mathrm{u}) \mu(\mathrm{v}) \neq 0$, where, $\Delta \mathrm{t}$ is the sampling period, and $\mathrm{N}_{\mathrm{w}}$ is the number of samples in the total duration of one poly-pulse waveform: $T_{m}+t_{p}$.

This CRB can be evaluated numerically, and is found to agree with the analytical expression in case of only additive noise [13]:

$$
\sigma_{v} \geq \frac{\lambda}{4 \pi \sqrt{2} \delta_{t}} \frac{\sqrt{C N R_{w f}+1}}{C N R_{w f}}
$$


in which $\mathrm{CNR}_{\mathrm{wf}}$ is the CNR taken in terms of waveform energy: $\mathrm{CNR}_{\mathrm{wf}}=\mathrm{E}_{\mathrm{waveform}} /\left(\mathrm{PSD}_{\text {noise }} \cdot \mathrm{t}_{\mathrm{p}}\right)$ ( $\mathrm{E}_{\text {waveform: }}$ waveform energy, $\mathrm{PSD}_{\text {noise: }}$ noise power spectral density).

As described in [7], error saturation and fading have to be taken into account. The resulting error after fading is indeed the quadratic sum of the CRB at given CNRs weighted by the probability of this CNR occurring (Rayleigh law). This probabilistic inclusion of fading helps better predict the error, but may affect the lower bound property.

Fig. 3 shows velocity error as a function of CNR with PP, PPP and ML estimators and CRBs on simulated signals assuming a non-vibrating target and a $5 \mathrm{kHz}$ bandwidth speckle noise. We inject the real values of CNR and $\mathrm{B}_{\text {speckle }}$ in the ML estimator.

In pulse-pair mode, theory predicts a limit for the velocity precision at high CNR, due to the predominant complex multiplicative noise. PP processing on a simulated signal shows the expected plateau related to this phenomenon, but much higher than expected. For this reason, we can suspect PP processing may not be optimal.

For 6-pulse waveforms, Fig. 3 shows globally lower velocity error. At high CNR, the CRB does not saturate, and decreases, which is not the case with PPP processing, as decorrelation between pulses impacts the velocity estimation. On the other hand, ML processing follows the bound and achieves velocity precision of about $0.2 \mathrm{~mm} / \mathrm{s}$ instead of $0.5 \mathrm{~mm} / \mathrm{s}$ with PPP, and $2 \mathrm{~mm} / \mathrm{s}$ with pulse-pairs, at high CNR. Indeed, when the measurement is predominantly affected by complex multiplicative noise, i.e. at high CNR, the ML estimator performs better than PPP, for any value of $B_{\text {speckle. }}$

The ML estimator is robust to the $\mathrm{CNR}$ and $\mathrm{B}_{\text {speckle }}$ parameters. For signals with $\mathrm{CNR}=20 \mathrm{~dB}$ and $\mathrm{B}_{\text {speckle }}=5 \mathrm{kHz}$, and varying parameter inputs for the ML estimator, performance is not significantly affected unless $\mathrm{B}_{\text {speckle }}$ or $\mathrm{CNR}$ are misestimated by several $\mathrm{kHz}$ 
and tens of $\mathrm{dB}$ respectively. As the estimation of these parameters on the amplitude of the signal provides very accurate values compared to such requirements, in the conditions where the ML estimator is useful $\left(\mathrm{B}_{\text {speckle }}\right.$ of a few $\left.\mathrm{kHz}, \mathrm{CNR}>0 \mathrm{~dB}\right)$, no problem should arise from the strategy of pre-estimating noise parameters. If it should prove difficult in practice, we are still able to evaluate the covariance matrix of the noise, after a fast PPP demodulation of the signal that would allow us to reconstitute a vibration suppressed signal containing only noise.

Finally, we have investigated the modification of the ML estimator for laser phase noise incorporated in the signal model, using the autocorrelation predicted by [17] for a narrow linewidth laser [18]. In that case, we notice that while 6-pulse waveforms still bring general precision improvement over pulse-pairs, ML processing does not provide much smaller velocity error than PPP, except for predominant speckle noise as we have already seen, and moderate decorrelations (optical path difference well below laser coherence length). When the correlation time of the signal is under pulse separation $\mathrm{T}_{\mathrm{S}}$, the CRB quickly rises, and both PPP and ML estimators stick to that bound.

We conclude that the ML estimator is interesting to implement in order to make pulsed vibrometry less sensitive to decorrelation such as the one produced by target speckle. The remaining velocity error approaches the $\mathrm{CRB}$ when $\mathrm{CNR}$ is high, which indicates it is close to optimal for the signal model of Eq. 1. Noise parameters are required as inputs and can be previously estimated on the signal amplitude. The impact of laser phase noise cannot be mitigated as much, certainly because the correlation time of the signal easily falls below $\mathrm{T}_{\mathrm{S}}$. We must finally stress that deviations from the signal model are well known to affect ML estimators. For instance, high frequency vibrations cause the IF to drift even within the short duration $\mathrm{T}_{\mathrm{m}}$ of the poly-pulse, in which case ML processing as we designed it here will not perform as well. 
This preliminary qualification is however incomplete without a thorough comparison to other estimators from literature on actual vibration signals, which is presented in section 5 .

\subsection{Time-Frequency Representation in Pulsed Mode}

Another way of improving signal processing in pulsed vibrometry is to perform signal tracking on a Time Frequency Representation, in order to compensate for the small number of measurements. A great gain over non-tracking methods is expected. At low CNR, it will avoid outlying values of velocity due to temporarily weak signal. Also multiple components caused by several vibrating parts of the target could be separated. The downside is that TFR processing is more demanding in computation time and memory.

In pulsed mode, a TFR can be drawn for three estimators we proposed in this article. MF (radar matched filter), AFT (see Tab. 1) and the ML estimator all rely on the search of a frequency maximum, whether it is a spectrum or a log-likelihood function, for each received waveform. We propose to track the frequency localization of the maxima of energy for noise mitigation at low CNR. We build a $2 \mathrm{D}$ representation with, as coordinates, frequency and waveform number, which can be linked to instants in time. Fig. 4 shows examples of what can be obtained on simulated signals.

From a five-tone vibration with maximum velocity of about $6 \mathrm{~mm} / \mathrm{s}$, we simulate the heterodyne signal according to the model of Eq. 1. The average CNR (in $1 \mathrm{MHz}$ bandwidth) is chosen as low as $-20 \mathrm{~dB}$ to show the phenomenon of spurious peaks that come from temporarily strong noise or weak signal, and strong speckle noise of bandwidth $\mathrm{B}_{\text {speckle }}=5 \mathrm{kHz}$ is applied. 6pulse waveforms with $t_{p}=2 \mu \mathrm{s}, \mathrm{T}_{\mathrm{S}}=50 \mu \mathrm{s}$ and $\mathrm{PRF}=500 \mathrm{~Hz}$ are used. For ML we choose a $1 / 3 \mathrm{~T}_{\mathrm{m}}$ frequency step, and for MF and AFT, which are based on Fast Fourier Transforms, zeropadding is applied to provide a similar resolution. Also, for better visibility, the plotted function 
is the normalized likelihood function, instead of the log-likelihood of Eq. 9. The functions for each waveform are plotted within ambiguity range $\left[-1 / 2 \mathrm{~T}_{\mathrm{S}} ; 1 / 2 \mathrm{~T}_{\mathrm{S}}\right]=[-10 \mathrm{kHz} ; 10 \mathrm{kHz}]$. On the left side of these charts, there is no additional processing. The frequency modulated signal is seen as a discontinuous trace, which produces a very noisy velocity time series if processed by maximum picking or even by centroiding.

The interest of TFR is highlighted here, as the correct vibration trace is still visible, despite isolated peaks originating from the noise, whereas standard algorithms give velocity time series corrupted by many outliers. We use an IF continuity hypothesis on the pseudo TFR in order to better extract the vibration in case of strong noise conditions, which should especially benefit our ML processing, inherently inclined to be affected in such a case.

A number of techniques are available that take advantage of the continuity of the signal trace $[19,20]$. We rather choose a simple temporal smoothing of the TFRs, as is done from on the right side of Fig. 4. The lines of the TFR have been low-pass filtered, with a bandwidth preserving the higher frequencies of vibration. If a few consecutive waveforms detect slowly drifting IF, due to the frequency spread the energy is integrated, whereas transitory peaks from the noise are smoothed. Such regularization allows a better recovery of the vibration velocity time series by a subsequent maximum picking or centroiding, especially in the case of ML processing, as will be seen in the next section.

In our comparative simulations of processing methods and waveforms, we applied temporal smoothing to MF, AFT and ML generated TFRs, and also to TFRs in continuous mode, for a fair comparison. The methods are then annotated accordingly: MF-s, AFT-s, ML-s, SGC-s, LSM-s. 


\section{Comparative simulation of processing methods in pulsed and CW mode}

We now study the performance of the advanced processing techniques that were introduced in section 4 , relative to the existing methods presented in section 3 . This comparison is conducted on realistic signals, with additive detection noise and complex multiplicative speckle noise, with a vibration comprising several modes. In this section, the study is based on simulated signals.

The simulation relies on the model developed in section 2. Subroutines generate the complex phasor induced by the vibration, as well as additive and multiplicative noises, which are re-sampled as suitable for each type of waveform, and used to produce the signal. The same mean power hypothesis is taken into account as a normalization of signal amplitude.

Using simulated heterodyne signals, we qualify all presented processing methods on the criterion of Signal-to-Noise Ratio (SNR), which is evaluated on the vibration spectrum, as the ratio of the Power Spectral Density (PSD) at the peak frequency of interest over the power spectral density of the noise floor. The PSD is estimated using the periodogram. The simulated vibration has 5 peak frequencies between 8 and $120 \mathrm{~Hz}$, and the maximum velocity is over $5 \mathrm{~mm} / \mathrm{s}$. The retained SNR value is the average of the 5 individual SNRs. For each given result, we average the SNR values obtained in 200 signal realisations.

The wavelength is $\lambda=1.55 \mu \mathrm{m}$; measurement duration is $\mathrm{T}_{\mathrm{mes}}=1 \mathrm{~s}$, with sampling frequency $\mathrm{f}_{\mathrm{ech}}=1 \mathrm{MHz}$ in $\mathrm{CW}$, and $2 \mathrm{MHz}$ in pulsed mode. In pulsed mode, the modulation parameters are: $\mathrm{N}_{\mathrm{p}}=2$ (pulse-pairs) or $\mathrm{N}_{\mathrm{p}}=6$ (6-pulse), $\mathrm{t}_{\mathrm{p}}=2 \mu \mathrm{s}, \mathrm{T}_{\mathrm{S}}=50 \mu \mathrm{s}$ and $\mathrm{PRF}=500 \mathrm{~Hz}$, which is a little above Nyquist's criterion for the correct sampling of the vibration. The analysis bandwidth in which the IF estimation is performed, both in CW and pulsed mode, is $\mathrm{B}_{\mathrm{a}}=20 \mathrm{kHz}$, corresponding to $\pm 7.75 \mathrm{~mm} / \mathrm{s}$ at our working wavelength. $\mathrm{B}_{\mathrm{a}}$ is thus closely adapted to the signal 
bandwidth induced by vibration and speckle. The spectrogram and autocorrelation window duration in $\mathrm{CW}$ mode is chosen as $1 / \mathrm{PRF}=2 \mathrm{~ms}$. The CNR is still given in average in a $1 \mathrm{MHz}$ bandwidth for $\mathrm{CW}$ mode (noted $\langle\mathrm{CNR}\rangle$ ); for comparison, the peak CNR in pulsed mode, in the reduced analysis bandwidth, is greater by $17 \mathrm{~dB}(6 \mathrm{p})$ or $27 \mathrm{~dB}(2 \mathrm{p})$. The speckle noise bandwidth is $\mathrm{B}_{\text {speckle }}=5 \mathrm{kHz}$.

Fig. 5 shows vibration spectra averaged over 200 runs, obtained at high CNR: $30 \mathrm{~dB}$. Only the best results for each waveform are plotted. The 5 vibration peak frequencies are visible on each curve, and can be identified. However, the noise floor is much lower with poly-pulse waveforms and ML estimator with smoothing (ML-s), or with CW and SGC-s, than with pulsepairs, which remain the most sensitive to the strong speckle noise applied in this simulation. In that case, pulse-pair operation cannot detect vibration modes with peak velocities under 0.1 $\mathrm{mm} / \mathrm{s}$, contrarily to poly-pulses with advanced processing.

A more detailed comparison in terms of SNR results for all processing methods is presented in Fig. 6 a) at high CNR, and in Fig. 6 b) at very low CNR. The mean SNR of each method is plotted as well as its standard deviation over the 200 runs.

At high CNR $(<\mathrm{CNR}\rangle=30 \mathrm{~dB})$, when speckle noise is predominant, all processing methods in CW mode perform equivalently well, whereas important differences appear between the various techniques applied to pulsed mode. First, pulse-pair SNR is more than 10dB worse than the best results obtained with 6-pulse waveforms or in $\mathrm{CW}$ mode. Better averaging of the phase noise with "longer" waveforms is the reason of such difference. We also find an average 5dB higher SNR given by the ML estimator proposed here, compared to the other estimators, including the commonly used radar matched filter (MF). However, it should be noted that CNRs over $15 \mathrm{~dB}$ are scarcely encountered, and in practice this gain may remain limited (see section 6). 
The PPP estimator offers relatively good performance (on top of low computational load), because it only relies on pairs of consecutive samples, which have less chance of being decorrelated, whereas standard spectral estimators look for a global phase trend, more easily corrupted when ambiguities are possible.

At very low CNR $(<\mathrm{CNR}\rangle=-25 \mathrm{~dB}$ in $1 \mathrm{MHz})$, it is known that without speckle noise high peak power waveforms (such as pulse-pairs) allow slightly better SNR on the measured vibration spectrum. In this simulation, however, because speckle noise is strong, there is no such gain. In CW mode, spectrogram centroiding allows the best SNR, while Lee's spectral matching is badly affected by strong detection noise. With poly-pulse waveforms, all estimators are roughly equivalent. Temporal smoothing benefits more to spectrogram processing in $\mathrm{CW}$ (5dB gain) than to ML and MF processing with poly-pulses (3dB gain). This is because of the lesser number of averaged columns of the pseudo TFR in pulsed mode. We also note that ML and MF are equivalent when the effects of speckle noise are not predominant.

The results obtained at medium CNR are intermediary. The usual plateau at higher CNR starts at $\mathrm{CNR}_{\mathrm{CW}, 1 \mathrm{MHz}}=-5 \mathrm{~dB}$. However ML performance does not stall and increases slowly with CNR.

The computational load of the various processing methods is summarized in Tab. 2. Attention is called to the following results: pulse-pair based estimators are practically immediate compared to all other estimators, and can provide an initial estimate of the vibration velocity, for more complex estimators that would be employed afterward. The ML estimator is the fastest spectral estimator used here. MF is equivalently fast. SGC remains the best but also the heaviest estimator in this study. As expected, pulsed vibrometry is faster to process in general, due to the fewer number of samples. 
We now conduct another performance simulation with a varying analysis bandwidth $B_{a}$, that was previously fixed at $20 \mathrm{kHz}$ to match the vibration bandwidth. This assumption was in fact very restrictive because, in practice, the bandwidth of the vibration is unknown, so $T_{S}$ and thus $B_{a}=1 / T_{S}$ have to be chosen large enough so that no velocity ambiguity is possible. Yet, bandwidth adaptation is important at low CNR, so as to avoid letting too much additive detection noise in the IF estimators.

All other simulation parameters remaining the same, in Fig. 7, we plot SNR results obtained at low CNR (-20dB), when bandwidth adaptation matters the most, as a function of bandwidth adaptation parameter $\alpha=B_{a} / B_{v i b}$, where $B_{\text {vib }}$ is the vibration induced frequency excursion. It shows that SNR is very dependent of the analysis bandwidth. When $\alpha<1$, as expected, signal losses and Doppler ambiguities deteriorate the measurement, especially in pulsed mode, for which $\alpha$ slightly above one is preferable to avoid ambiguities. But for $\alpha>1$, the various estimators react differently to the more important noise accepted in the analysis bandwidth. Temporal smoothing in both $\mathrm{CW}$ and pulsed mode is necessary to avoid a fall of SNR beyond $\alpha=2$. For instance, for a $100 \mathrm{kHz}$ analysis bandwidth, i.e. $\mathrm{T}_{\mathrm{S}}=10 \mu$ s and $\alpha=5$, only pulsed mode with poly-pulse waveforms processed by ML or MF and pseudo TFR temporal smoothing is able to retain around $10 \mathrm{~dB}$ SNR. In some conditions, even with strong speckle noise, pulsed mode as enhanced by the methods described in this article can be preferable, because of its robustness to additive noise.

\section{Experimental validation}

\subsection{Apparatus}


In order to confirm the results of section 5 , an all-fiber $1.55 \mu \mathrm{m}$ vibrometer was implemented, with the configuration described in Fig. 8. Fibered systems with a MOPA configuration are an interesting choice in this case, because of their compactness, ease of use, and versatility; switching from $\mathrm{CW}$ emission to pulsed emission only requires applying a modulation on the driving signal of the Acousto-Optic Modulator (AOM) used for the frequency shifting of the emitted laser wave.

Separate optics were used for emission and reception, which is essential in CW mode on static targets. The output waveforms were monitored because the amplifier distorts the input impulsions, and the modulating signal needs to be adjusted in compensation. The laboratory experiment also includes an apparatus to create the same vibration as the one used in section 5,

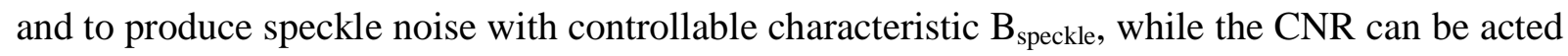
upon via a tunable attenuator placed before the emission optics.

The optical beam from a Koheras laser injector delivering $10 \mathrm{~mW}$ with $90 \mathrm{kHz}$ linewidth is split into a signal beam and a LO beam. The signal wave is modulated by an IntraAction AOM frequency shifter at $70 \mathrm{MHz}$. Its driving signal can be amplitude modulated in pulsed mode by a digital gating signal that provides the waveform frame and an analogic signal, generated by a Wavetek arbitrary waveform generator, which allows the fine tuning of the emitted power along time. Both signals are necessary to ensure correct extinction of the emission. The modulated optical wave is then fed into a Keopsys 1W Erbium Doped Fiber Amplifier (EDFA), operated at $100 \mathrm{~mW}$ output. The power is sent to the emission telescope, but can be attenuated to vary the CNR. The emitted wave reflects on the vibrating mirror, a few meters away, and is scattered by a rotating diffuse target. The rotation rate of the target sets the speckle bandwidth $\mathrm{B}_{\text {speckle. }} \mathrm{A}$ 
second telescope receives this backscattered wave, which is mixed with the LO on a Hamamatsu InGaAs detector with a $100 \mathrm{MHz}$ bandwidth.

The heterodyne signal around $70 \mathrm{MHz}$, is amplified and frequency downshifted by mixing it with the $67.5 \mathrm{MHz}$ output of a HF generator. The resulting signal, with $2.5 \mathrm{MHz}$ intermediate frequency, is low-pass filtered at $5 \mathrm{MHz}$ and digitized on 16 bits at a $15 \mathrm{MHz}$ sampling frequency, by a National Instruments 5922 acquisition card (ADC).

Signal processing is then implemented on Matlab, for 1 second duration acquisitions. The analytic signal (complex signal around null frequency) is derived by a Hilbert transform of the acquisition, and is either decimated to $1 \mathrm{MHz}$ sampling frequency in $\mathrm{CW}$ mode, or pulseaveraged in pulsed mode. Both of these methods allow useful data reduction. Finally, the processing methods discussed in this study are applied to determine the vibration spectrum of the mirror, and performance in terms of SNR is calculated by the same means as in section 5 .

Parameters $t_{p}, T_{S}$ and PRF of the waveforms are also roughly identical to the ones chosen previously. As expected, the amplitude modulation applied on the AOM driving signal has to be pre-compensated in order to obtain a suitable waveform amplitude function $\mu(t)$, because of EDFA-related phenomena [21]. In order to avoid having to retune this pre-compensation as we switch from one waveform type to another, pulse-pair mode is implemented by simply taking the first two pulses of 6-pulse waveforms. After a preliminary calibration of average CNR in regard to output power in the several emission modes, we vary the power attenuation at the emission in order to study the SNR given by each estimator at various CNR values. The speckle bandwidth induced by the rotating disk is set to approximately $5 \mathrm{kHz}$, as verified by fitting the autocorrelation function of the signal, without vibration. 
Two main difficulties had to be resolved. In pulsed mode, we had to overcome the saturation of the electrical amplification chain for high signal peak power, which originally saturated the CNR at 10dB. Also, parasitic vibrations of the speckle generating disk (including a broadband component that particularly impacted on the ML estimator, which assumes stationary IF during the waveform) had to be minimized, which was done by using lower rotation rates and larger spot sizes and thus a larger vibrating mirror. These parasitic vibrations were reduced by a factor of 2 but could not be entirely suppressed; the following experimental results include this artifact.

\subsection{Results}

Fig. 9 summarizes the results of this experimental comparison of waveforms and signal processing methods, with a) SNR vs CNR curves for the best methods found in the previous section and b) average vibration spectra at high CNR.

Fig. 9 b) shows the vibration spectra averaged on 20 to 40 runs of 1 second each, at the highest CNR achieved for each waveform. We see the vibration is fine-tuned to be almost identical to that used in the simulations (cf. Fig. 5).

The SNR vs CNR curves on Fig. 9 a) follow the typical increasing trend with a plateau at high CNR, when velocity precision becomes speckle-limited. However, parasitic vibrations cause of this SNR saturation to occur about 3dB lower than in simulation. At low CNR, we find the expected SNR fall as $\mathrm{CNR}^{2}$ for spectrogram demodulation (SGC) and as CNR for pulse-pair vibrometry (PP), as demonstrated in [7].

Overall, the simulation results are corroborated, including the better performance of the ML estimator at high CNR, compared to more classical PPP or MF processing. 6-pulse waveforms are clearly more successful than pulse-pairs in these conditions. This is also seen by 
comparing the vibration spectra obtained at high CNR on Fig. 9 b). However, experimental artifacts make the gain in SNR of the ML estimator less important than in simulation. It is also noticeable that this gain does not become interesting unless the CNR is above $5 \mathrm{~dB}$, and after that threshold it increases linearly, as simulations showed. Unfortunately, as CNR over $20 \mathrm{~dB}$ is difficult to obtain, very important gains due to the use of ML over regular IF estimators cannot be relied upon in practice.

Yet, the SNR increase due to TFR smoothing, which nears 5dB in some cases, was not expected to persist at such high CNR values in pulsed mode. It is still measurable when signal is fairly strong, which means that the impact of signal fading is underestimated in our simulation, and that TFR smoothing is indeed helpful in pulsed mode, when velocity measurements are few.

\section{Conclusion}

We have presented and qualified advanced signal processing techniques applicable to pulsed laser vibrometry with poly-pulse waveforms, to enhance the determination of the modal frequencies of a remote target in harsh noise conditions. This work focuses on what we believe is the first extensive comparison between these waveforms and processing methods, particularly in the case when this measurement is made difficult by strong decorrelation noise like target speckle noise. It can indeed reduce the interest of pulsed vibrometry, for instance when compared to classical $\mathrm{CW}$ vibrometry that averages numerous velocity measurements. An additional hypothesis is taken: an equal mean laser power to be distributed between emitted waveforms.

In this frame of work, poly-pulse waveforms do not have peak power as high as the first employed pulse-pairs, and will not present as much gain at very long range. But because of their longer effective duration when the pulse separation is set to avoid Doppler ambiguities, every 
test presented here has proved them more robust in the said noise conditions. Because it did not exist in pulsed mode, a specific processing based on Maximum Likelihood Instantaneous Frequency estimation, which takes into account what we know of the noise statistics, has been developed. By comparing the velocity precision of this processing method to its theoretical limits (CRB), it has been shown that it is closer to optimal than that of simple poly-pulse pair processing. Also, we have proposed Time-Frequency Representations, already applied in CW vibrometry, and show that they can be of use for noise regularization in pulsed vibrometry, for instance by temporal smoothing.

A comprehensive comparison of the SNR performance of the described processing to that of classical methods, for vibrometry with pulsed and CW emission, has been conducted on simulated signals, and later confirmed by experimentation. The first conclusion is that advanced processing, based on the TFR of the likelihood as a function of the IF for each waveform, with an additional temporal smoothing of this TFR, gives the best result in pulsed vibrometry, especially with dominant speckle, with no additional computational cost. Secondly, in case of weak signals (low CNR), at long range for instance, temporal smoothing of TFR proved useful as a noise regularization. Moreover, in case of mismatched analysis bandwidth, pulsed vibrometry with poly-pulse waveforms and matched filtering was finally shown to be more robust than CW vibrometry with spectrogram centroid demodulation. This is in spite of the strong speckle noise that was impacting the signal.

However, these results are tempered by the high CNR required for the developed estimator to obtain a gain in SNR, and its sensitivity to deviations from the model assumed in section 2 of this article. As has been proven by our experiment, high frequency vibrations are enough to decrease its performance, because it assumes stationary IF during the velocity 
measurement time $\mathrm{T}_{\mathrm{m}}$. This defect could nevertheless be acted upon by including the possibility of IF drift in the model. Another issue of the current study is that, by assuming the same average laser power for each emission mode, it allowed a general comparison, but failed to take into account technological limitations. For instance, fiber lasers at $1.55 \mu \mathrm{m}$ with a MOPA configuration, such as the one we used, are instead limited by the peak power, or the energy accumulated in the amplifying fiber. A direct sequel of this study will be to evaluate the actual optimal performance of such a fibered vibrometer.

The results of this work still push towards the use of longer waveforms, of estimators that take into account signal statistics, and of TFR regularization in pulsed vibrometry. Further advances can be made in each of these three directions. With sufficiently resilient ambiguity resolving algorithms, and assuming suitable emission architecture, staggered poly-pulses can achieve very large measurement dynamics. Also, ML based parametric estimators including a complete model of the vibration remain the optimal processing method, if the computational load is not prohibitive despite the many parameters required for it to be robust. Finally, if the latter improvements are not possible, the vibration can still be better estimated from a TFR, by taking advantage of the signal continuity in the Time-Frequency space.

Our future works will involve studying such techniques for IF tracking on TFRs, and developing parametric estimation for other applications of vibrometry.

\section{Acknowledgements}

We thank Christopher Hill from QinetiQ, as well as Matthieu Valla and Guillaume Canat from Onera, for useful discussions, and Didier Goular and Béatrice Augère from Onera for technical support. This work was partly supported by the Délégation Générale de l'Armement, Mission pour la Recherche et l'Innovation Scientifique. 


\section{References}

1. P. Gueguen, V. Jolivet, C. Michel, A.-S. Schveitzer, "Comparison of velocimeter and coherent lidar measurements for building frequency assessment”, Bull. of Earthquake Engin. 8, 327-338 (2010).

2. W. Kranz, "Target Classification by Laser Vibration Sensing”, Proc. SPIE 1181, 301-306 (1989).

3. S. M. Hannon, J. A. Thomson, S. W. Henderson, P. Gatt, R. Stoneman, D. Bruns, “Agile Multiple Pulse Coherent Lidar for Range and Micro-Doppler Measurement”, Proc. SPIE 3380, 259-269 (1998).

4. P. Gatt, S. W. Henderson, B. Krause, "Poly-pulse Coherent Lidar Waveforms for Coherent Lidar Measurements", Coh. Opt. Tech. and App. Conf., Whistler, Canada (2006).

5. S. W. Henderson, J. A. Thomson, S. M. Hannon, P. Gatt, "Comparison of Pulsed Waveform and CW Lidar for Remote Vibration Measurement", $10^{\text {th }}$ Coh. Laser Radar Conf., Mount Hood (1999).

6. C. Hill, M. Harris, K. D. Ridley, "Fiber-based $1.5 \mu \mathrm{m}$ lidar vibrometer in pulsed and continuous modes", App. Opt. 46, 4376-4385 (2007).

7. P. Gatt, S. W. Henderson, S. M. Hannon, "Noise Mechanisms Impacting Micro-Doppler Lidar Signals: Theory and Experiment”, Coherent Technologies, Inc. Technical Report (2000).

8. C. Hill, M. Harris, K. D. Ridley, E. Jakeman, P. Lutzmann, "Lidar frequency modulation vibrometry in the presence of speckle", App. Opt. 42, 1091-1100 (2003).

9. A. Ishimaru, "The Beam Wave Case and Remote Sensing”, in Topics in Applied Physics 25: Laser Beam Propagation in the Atmosphere, J. W. Strohbehn, ed (1978), pp. 129-170. 
10. A. L. Kachelmyer and K. I. Schultz, "Spectrogram Processing of Laser Vibration Data", Proc. SPIE 1936, 78-88 (1993).

11. D. G. Youmans, "Joint Time-Frequency Transform Processing for Linear and Sinusoidal FM Coherent Ladars", Proc. SPIE 5087, 46-57 (2003).

12. W. F. Buell, B. A. Shadwick, R. W. Farley, "Bayesian Spectrum Analysis for Laser Vibrometry Processing”, Institute for Advanced Physics Report (2000).

13. B. J. Rye, R. M. Hardesty, "Discrete Spectral Peak Estimation in Incoherent Backscatter Heterodyne Lidar”, IEEE Trans. Geoscience and Remote Sensing 31, 16-35 (1993)

14. D. G. Youmans, "Target Spectral Estimation using Direct Detection and Coherent Detection Ladar”, Proc. SPIE 5791, 97-108 (2005).

15. B. C. Lovell, R. C. Williamson, "The Statistical Performance of Some Instantaneous Frequency Estimators”, IEEE Trans. Sig. Proc. 40, 1708-1723 (1992)

16. M. Ghogho, A. K. Nandi, A. Swami, "Cramér-Rao Bounds and Maximum Likelihood Estimation for Random Amplitude Phase-Modulated Signals”, IEEE Trans. Sig. Proc. 49, 2905-2916 (2001).

17. J.-Ph. Tourrenc, Ph. Signoret, M. Myara, M. Bellon, J.-Ph. Perez, J.-M. Gosalbes, R. Alabedra, B. Orsal, “Low-Frequency FM-Noise-Induced Lineshape: A Theoretical and Experimental Approach”, IEEE Journ. Quant. Electr. 41, 549-553 (2005).

18. C. Spiegelberg, J. Geng Y. Hu, Y. Kaneda, S. Jiang, N. Peyghambarian, "Low-Noise Narrow-Linewidth Fiber Laser at 1550 nm”, IEEE Journ. of Lightwave Tech. 22, 57-62 (2004).

19. O. Michel, A. Hero, P. Flandrin, "Graphes de représentation minimaux, entropies et divergences: applications”, Traitement du Signal 17, 287-297 (2000). 
20. T. Thayaparan, L. Stankovic, I. Djurovic, S. Penamati, K. Venkataramaniah, "Intelligent target recognition using micro-Doppler radar signatures”, Proc. SPIE 7308, 17-28 (2009).

21. D. N. Schimpf and C. Ruchert, "Compensation of pulse-distorsion in saturated laser amplifiers", Opt. Express 16, 17637-17646 (2008). 


\section{Figures}

Fig. 1. (color online) Diagram of a heterodyne coherent lidar vibrometer, with a Master

Oscillator Power Amplifier (MOPA) configuration, convenient to emit arbitrary waveforms using the same Acousto-Optic Modulator (AOM) as for the frequency shift.

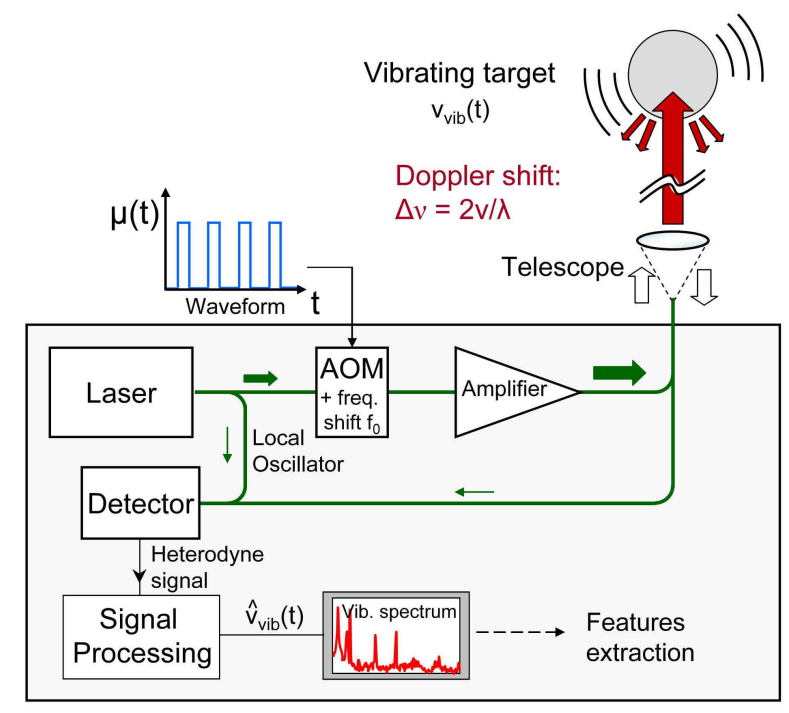


Fig. 2. (color online) Parameters of poly-pulse waveforms in amplitude modulation $\mu(t)\left(t_{p}\right.$ : pulse duration, $\mathrm{T}_{\mathrm{S}}$ : pulse separation, $\mathrm{T}_{\mathrm{m}}$ : poly-pulse duration, PRF: waveform repetition frequency, $\mathrm{N}_{\mathrm{p}}$ : pulse number per waveform, $\mu_{\max }$ : maximal amplitude of $\left.\mu(t)\right)$

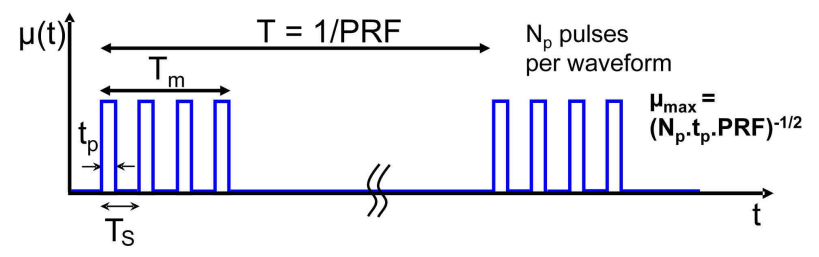


Fig. 3. (color online) Comparison of velocity errors of PP, PPP and Maximum Likelihood (ML) estimators and theoretical Cramér-Rao lower Bound (CRB) for the velocity error, as a function of average CNR, for pulse-pair (2p) and 6-pulse (6p). 2000 simulated waveforms with $\mathrm{T}_{\mathrm{S}}=50 \mu \mathrm{s}$ and $t_{p}=2 \mu \mathrm{s}$, at $B_{\text {speckle }}=5 \mathrm{kHz}$. Only the ML estimator reaches the CRB.

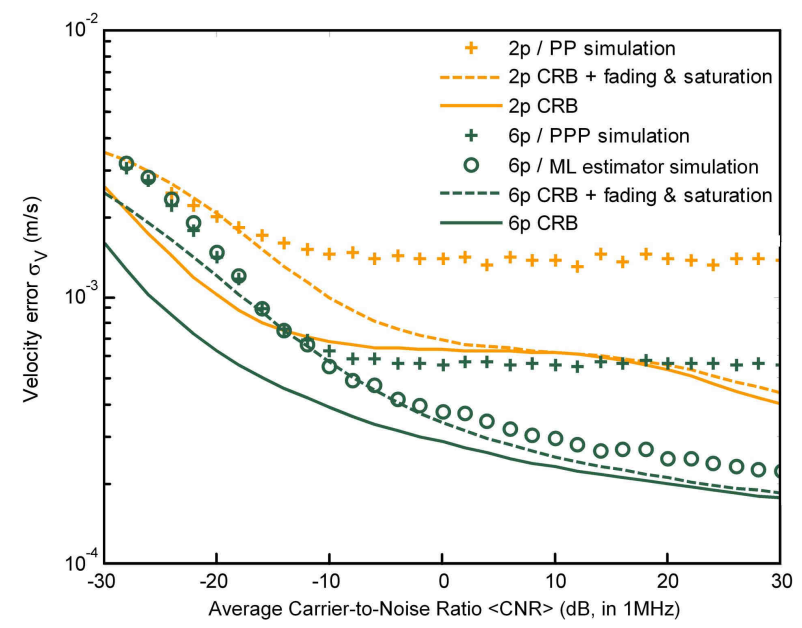


Fig. 4. Time-Frequency Representations (TFRs) obtained for simulated signals (6-pulse waveforms, $\mathrm{PRF}=500 \mathrm{~Hz}, \mathrm{~T}_{\mathrm{S}}=50 \mu \mathrm{s}$ and $\mathrm{t}_{\mathrm{p}}=2 \mu \mathrm{s}$ ) with matched filtering, autocorrelation Fourier Transform, and likelihood, at low CNR $(-20 \mathrm{~dB}$ in $1 \mathrm{MHz})$, and $\mathrm{B}_{\text {speckle }}=5 \mathrm{kHz}$. On the right side, temporal smoothing of the TFRs brings out the vibration trace.

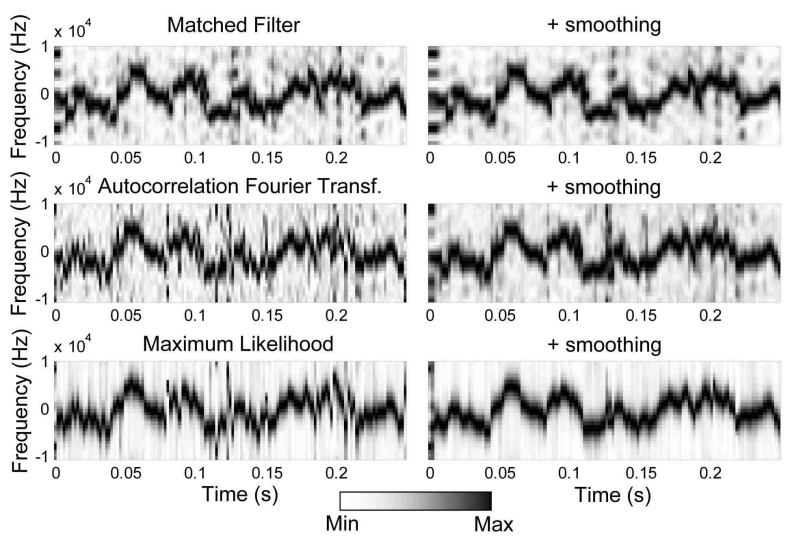


Fig. 5. (color online) Average vibration spectra simulated at high CNR (30dB in $1 \mathrm{MHz}$ ), processed by the estimators giving the best SNR for CW, pulse-pairs (2p) and 6-pulse waveforms (6p). The noise floor is much lower with the ML estimator than with the PPP estimator.

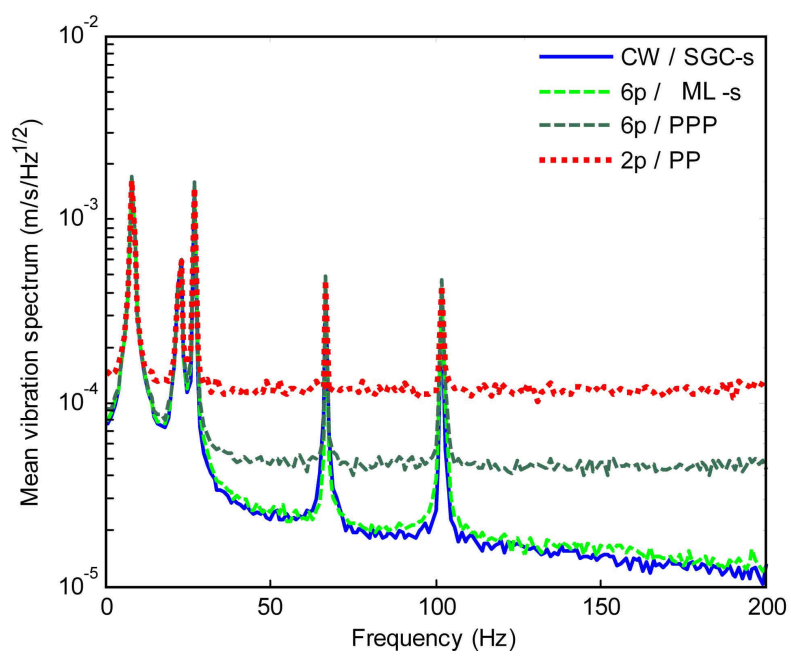


Fig. 6. Average $\mathrm{SNRs}$ obtained in simulation at $\mathrm{B}_{\text {speckle }}=5 \mathrm{kHz}$ and a) (top) high $\mathrm{CNR}$ (30dB) or b) (bottom) low CNR (-25dB) for CW, pulse-pairs (2p) and 6-pulse waveforms (6p). Suffix -s means that smoothing is applied on the pseudo TFR obtained using the preceding estimator.
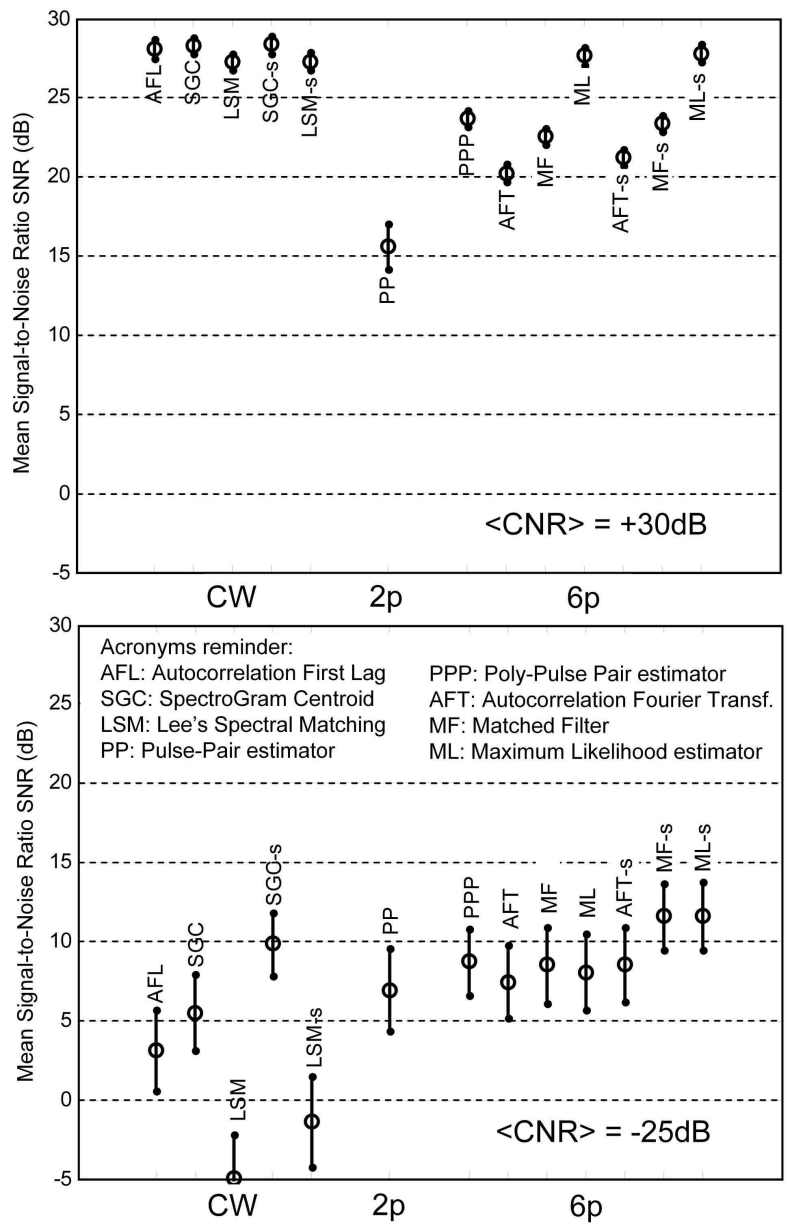
Fig. 7. (color online) Average SNRs at low CNR (-20dB) plotted as a function of the ratio between analysis bandwidth $B_{a}$ and vibration bandwidth $B_{\text {vib }}$, for $C W$, pulse-pairs (2p) and 6pulse waveforms (6p).

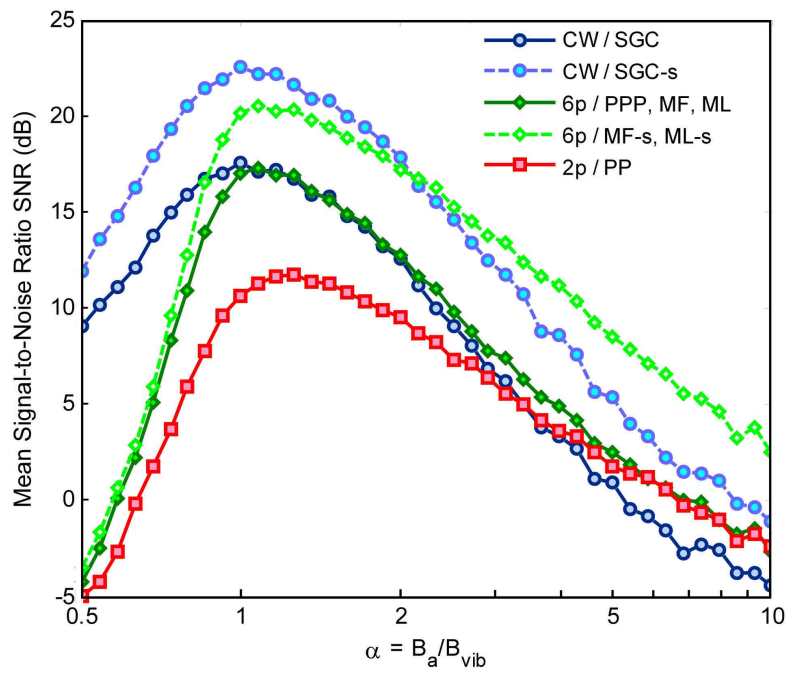


Fig. 8. (color online) Diagram of experimental apparatus allowing CW and poly-pulse operations. A rotating scatterer is used to create speckle noise with bandwidth $\mathrm{B}_{\text {speckle }}=5 \mathrm{kHz}$. (EDFA: Erbium-doped fiber amplifier, ATT: attenuator, Det: detector, HF: high frequency, ADC: analog to digital converter).

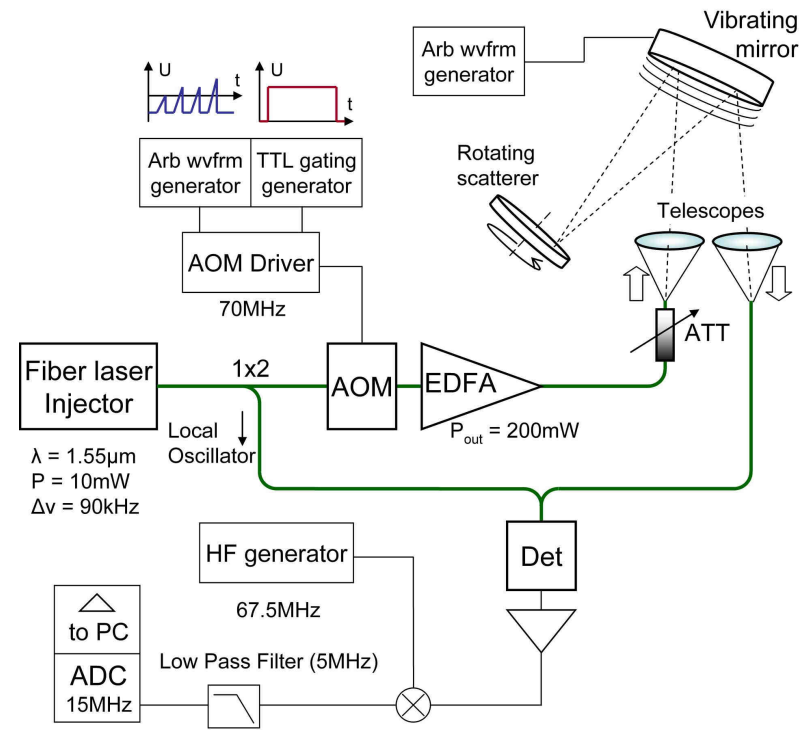


Fig. 9. (color online) a) (top) Experimental SNR plotted as a function of CNR and b) (bottom) Experimental spectra at high CNR, averaged over 20 to 40 measurements, for $\mathrm{CW}$, pulse-pairs (2p) and 6-pulse waveforms (6p).
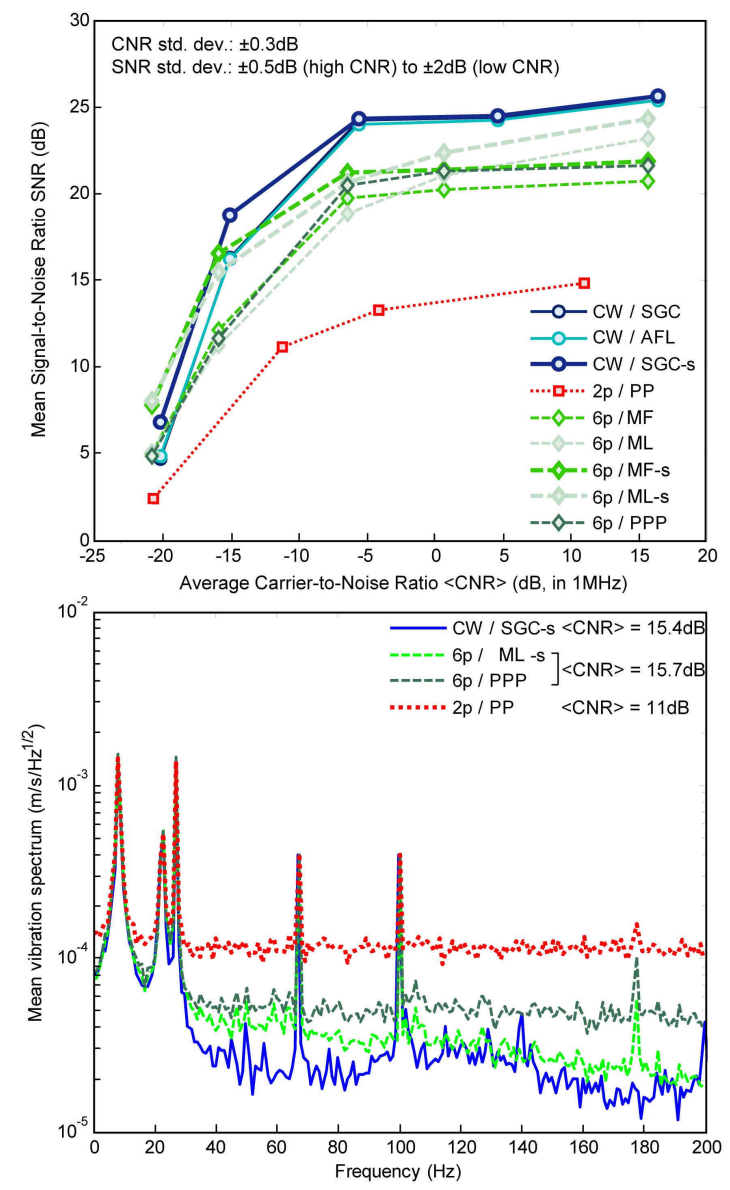


\section{Tables}

Table 1. Implemented instantaneous frequency estimators

\begin{tabular}{|c|c|c|c|c|}
\hline \multirow{3}{*}{$\begin{array}{l}\stackrel{8}{8} \\
\stackrel{0}{a} \\
3 \\
己\end{array}$} & AFL & $\hat{f}_{i n s t}(t)=\frac{1}{2 \pi \Delta t} \arg \left(\sum_{n=1}^{N_{m}} i_{s}(t+n \cdot \Delta t) i_{s}^{*}(t+(n-1) \cdot \Delta t)\right)$ & (2) & $\begin{array}{l}\Delta \mathrm{t}: \text { sampling } \\
\text { period, } \mathrm{n}: \\
\text { sample index, } \\
\mathrm{N}_{\mathrm{m}} \text { : number of } \\
\text { samples for IF } \\
\text { estimation }\end{array}$ \\
\hline & SGC & $\hat{f}_{\text {inst }}(t)=\frac{B_{a}}{2 \pi} \arg \left(\int_{B a}|\operatorname{STFT}(t, f)|^{2} \cdot \exp \left(j 2 \pi \frac{f}{B_{a}}\right) d f\right)$ & (3) & $\begin{array}{l}\mathrm{B}_{\mathrm{a}}: \text { analysis } \\
\text { bandwidth, } \\
|\mathrm{STFT}(\mathrm{t}, \mathrm{f})|^{2}: \\
\text { spectrogram }\end{array}$ \\
\hline & LSM & $\hat{f}_{\text {inst }}(t)=\underset{f}{\arg \max }\left(S_{\text {ref }}(f) \otimes|\operatorname{STFT}(t, f)|^{2}\right)$ & (4) & $\begin{array}{l}S_{\text {ref }}(f) \text { : reference } \\
\text { spectrum }\end{array}$ \\
\hline \multirow{4}{*}{ 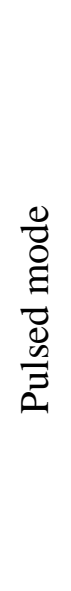 } & PP & $\hat{f}_{\text {inst }}(t=k / P R F)=\frac{1}{2 \pi T_{S}} \arg \left(<i_{s}>_{k, 2}<i_{s}>_{k, 1}^{*}\right)$ & (5) & \multirow{2}{*}{$\begin{array}{c}\mathrm{k} \text { : waveform } \\
\text { index, }\left\langle\mathrm{i}_{\mathrm{s}}\right\rangle_{\mathrm{k}, \mathrm{p}} \\
\text { signal average } \\
\text { over pulse \#p of } \\
\text { waveform \#k }\end{array}$} \\
\hline & PPP & $\hat{f}_{\text {inst }}(t=k / P R F)=\frac{1}{2 \pi T_{S}} \arg \left(\sum_{p=2}^{N_{p}}\left\langle i_{s}\right\rangle_{k, p}<i_{s}>_{k, p-1}^{*}\right)$ & (6) & \\
\hline & AFT & $\hat{f}_{\text {inst }}(t=k / P R F)=\underset{f}{\arg \max }\left(\int \arg \left(\Gamma_{i_{s}, k}(\tau)\right) \cdot h(\tau) \cdot \exp (-j 2 \pi f \tau) d \tau \mid\right)$ & (7) & $\begin{array}{c}\Gamma_{\mathrm{is}, \mathrm{k}}(\tau): \\
\text { autocorrelation } \\
\text { of poly-pulse } \\
\# \mathrm{k}, \mathrm{h}(\tau) \text { : } \\
\text { weighting } \\
\text { function } \\
\end{array}$ \\
\hline & MF & $\hat{f}_{\text {inst }}(t=k / P R F)=\underset{f}{\arg \max }\left(\left|\int_{\text {Poly-pulse \#k }} i_{s}\left(t^{\prime}\right) \cdot \mu\left(t^{\prime}\right) \cdot \exp \left(-j 2 \pi f t^{\prime}\right) d t^{\prime}\right|\right.$, & (8) & $\begin{array}{l}\text { Radar matched } \\
\text { filter }\end{array}$ \\
\hline
\end{tabular}

Acronyms definition: AFL: Autocorrelation First Lag, SGC: SpectroGram Centroid, LSM: Lee's

Spectral Matching, PP: Pulse-Pair, PPP: Poly-Pulse Pair, AFT: Autocorrelation Fourier

Transform, MF: Matched Filter. 
Table 2. Computation times for implemented estimators

\begin{tabular}{ccccccccc}
\hline Method & AFL & SGC & LSM & PP & PPP & AFT & MF & ML \\
\hline Comp. time (s) & 0.9 & 1.5 & 1.1 & $10^{-3}$ & $10^{-3}$ & 0.97 & 0.18 & 0.17 \\
\hline
\end{tabular}

These results are obtained on Matlab with a $2.13 \mathrm{GHz}$ CPU. 